\title{
ON THE AUTOMORPHISM GROUP OF COMPACT MEASURE HYPERBOLIC MANIFOLDS AND COMPLEX ANALYTIC BUNDLES WITH COMPACT MEASURE HYPERBOLIC FIBRES
}

\author{
B. WONG
}

\begin{abstract}
We prove the automorphism groups of the compact measure
\end{abstract} hyperbolic manifolds are discrete.

1. Introduction. The aim of this note is to study the open problem: Given a compact measure hyperbolic manifold, is the automorphism group finite? (See [1, Problem 11].) Although we cannot prove or disprove this conjecture, the following theorem is quite easy to show.

THEOREM 1. Given a compact measure hyperbolic manifold in the sense of Eisenman-Kobayashi, then its automorphism group is discrete.

It is known that a compact manifold of general type must be measure hyperbolic, but the converse is an open problem. The finiteness of the automorphism group of a compact manifold of general type is a well-known fact. (See [2] for a differential geometric proof.) Our conjecture would be settled if one could prove the automorphism group is compact. The compactness would follow from the existence of an invariant metric that induces the underlying topology; it is unknown even if we assume the manifold to be projective algebraic.

As an application of above result, we can prove the following theorems, which might be regarded as a generalization of recent results of $\mathbf{H}$. L. Royden [3].

THEOREM 2. (1) A holomorphic fibre bundle with simply connected base and compact measure hyperbolic fibres is holomorphically trivial.

(2) A holomorphic fibre bundle with compact measure hyperbolic fibre is a flat bundle.

We also obtain the following nontrivial fact as a consequence of Theorems 1 and 2. For some related results one can consult a recent survey article of Kobayashi (Intrinsic distance, measure and geometric function theory, Bull.

Received by the editors January 13, 1976 and, in revised form, April 12, 1976.

AMS (MOS) subject classifications (1970). Primary 32H15, 32H20; Secondary 32H25, 32A15.

Key words and phrases. Measure hyperbolic, automorphism group, complex analytic bundle. 
Amer. Math. Soc. 82 (1976), 357-416, Theorems 3.3 and 3.4).

THEOREM 3. A holomorphic fibre bundle with compact measure hyperbolic fibres and measure hyperbolic base is also measure hyperbolic.

ACKNowledgement. I am very grateful to express my gratitude to Professor S. Kobayashi for many helpful conversations during my stay in Berkeley.

2. Definitions and proof of Theorem 1 . Let $M$ be a complex manifold, $B_{n}$ the unit ball in $C^{n}$, and $V_{n}$ the volume form of the Bergmann metric in $B_{n}$.

The Eisenman-Kobayashi measure is defined as follows.

With respect to the local coordinates around a fixed point $P \in M$, one can write $K_{M}=k_{M} d z_{1} \wedge d \bar{z}_{1} \cdots d z_{n} \wedge d \bar{z}_{n}$, where

$k_{M}(P)=\inf \left\{|g(0)| \mid f^{-1}\left(d z_{1} \wedge d \bar{z}_{1} \cdots d z_{n} \wedge d \bar{z}_{n}\right)=g(x) \cdot V_{B}\right.$,

$$
\left.f \text { belongs to } M\left(B_{n}\right) \text {, and } f(0)=P\right\}
$$

(x belongs to $B_{n}$ ).

Definition. $M$ is measure hyperbolic if $K_{M}$ is nonzero almost everywhere (i.e. outside the union of finitely many complex analytic varieties).

REMARK. It is easy to see that the measure on $C \times F$, where $F$ is any complex manifold and $C$ is the complex line, is identically zero. The measure also possesses the important property that it is volume-decreasing under holomorphic mappings.

Proof of Theorem 1. Let us write $A=$ Aut $(M, M)$. Then $A$ is a complex Lie group acting holomorphically on $M$ by a famous theorem of Bochner and Montgomery. Suppose $A$ is not discrete. Let $V$ be a nonzero element of the Lie algebra of $A$ and $\exp (t V)$ the complex one parameter group acting in the direction of $V$. Then by a theorem of Bochner and Montgomery again, $\exp (t V)$ induces a nontrivial holomorphic vector field on $M$.

Since $M$ is measure hyperbolic, $K_{M}$ is nonzero outside the union $S$ of finitely many complex analytic varieties. The vector field cannot vanish identically on $M-S$, so we can choose $m \in M$ in such a way that both the holomorphic field and $K_{M}$ are not zero in a neighborhood of $m$.

Let $D_{n-1}$ be an $(n-1)$-dimensional complex analytic disc embedded in $M$ with center at $m$, where $D_{n-1}$ is transversal locally to the orbit of $\exp (t V)$ near $m$. Then $D_{n-1} \times \exp (t V)$ is contained in $M \times A$ in a natural way and so induces a holomorphic mapping $F: D_{n-1} \times C \rightarrow M$, such that $d F \neq 0$ close to $m$. However, the Eisenman-Kobayashi measure is identically zero in $D_{n-1} \times C$. By the volume-decreasing property, $K_{M}$ must vanish in a small neighborhood of $F(m)$. This is an obvious contradiction of the assumption that $M$ is measure hyperbolic.

3. Proofs of Theorems 2 and 3. (A) Every holomorphic fibre bundle with base $B$ and fibre $M$ can be described as a cocycle on $B$ with values in $A=$ Aut $(M, M)$ : There exists an open covering $\left\{U_{i}\right\}$ of $B$ such that the 
transition functions $f_{i j}: U_{i} \cap U_{j} \rightarrow A$ obey the cyclic rule $\left(f_{i j}\right) \cdot\left(f_{j k}\right)=\left(f_{i k}\right)$ on $U_{i} \cap U_{j} \cap U_{k}$, for all $i, j, k$. But $A$ is discrete, and $U_{i}$ can be chosen to be connected; hence $f_{i j}$ is constant on $U_{i} \cap U_{j}$. This is the standard definition of a flat bundle.

(B) It is a well-known fact that the flat bundles can be constructed from homomorphisms of the fundamental group of $B$ into $A=$ Aut $(M, M)$ (see Gunning, Lectures of vector bundles over Riemann surfaces, Princeton Univ. Press). If $B$ is simply connected, then the representation is trivial, so that the bundle is holomorphically equivalent to $M \times B$.

(A) and (B) together prove Theorem 2.

(C) Theorem 3 follows from the following two facts:

1. Let $B^{\prime}$ be the universal covering space of $B$. Then $B^{\prime}$ is measure hyperbolic iff $B$ is measure hyperbolic.

2. The product of two measure hyperbolic manifolds is also measure hyperbolic.

One observes that $M \times B^{\prime}$ covers the bundle with fibre $M$ and base $B$; our theorem follows immediately from the two assertions above.

\section{REFERENCES}

1. S. Kobayashi, Some problems on intrinsic distances and measures (preprint, Problem 11).

2. - Transformation groups in differential geometry, Ergebnisse Math. Grenzgebiete, Band 70, Springer-Verlag, Berlin and New York, 1972. MR 50 \#8360.

3. H. L. Royden, Holomorphic fiber bundles with hyperbolic fiber, Proc. Amer. Math. Soc. 43 (1974), 311-312. MR 49 \#3229.

Department of Mathematics, University of Toronto, Toronto, Canada M5S iAl 